\title{
USO DE LOS BIOMARCADORES EN LA EVALUACIÓN DE LA CONTAMINACIÓN
}

\author{
TORO-RESTREPO, B. ${ }^{1}$
}

beatriz.toro@ucaldas.edu.co

Manizales, 2010-04-29 (Rev. 2010-12-14)

\section{RESUMEN}

Cada día es más difícil evaluar la salud de un ecosistema debido a la gran cantidad y diversidad de xenobióticos que son introducidos en ellos. Es por eso, que en las últimas dos décadas se ha incrementado el uso de biomarcadores para la evaluación y monitoreo de los ecosistemas. Un biomarcador es una respuesta funcional, fisiológica o bioquímica al nivel celular o interacciones moleculares que un organismo, una población o una comunidad refleja frente a un peligro potencial, el cual puede ser químico, físico o biológico. El biomarcador representa una señal integrada del nivel de contaminación de una determinada zona y, por consiguiente, constituye un indicador del nivel de riesgo toxicológico al que puede ser sometida una determinada población natural. No obstante, para que sea efectiva una evaluación ambiental o un programa de biomonitoreo, se deberán utilizar múltiples biomarcadores que describan los síntomas que sugieren la relación causa-efecto para una especie.

\section{PALABRAS CLAVE:}

Biomarcadores, respuestas fisiológicas, ecotoxicología, contaminación, monitoreo.

\section{USE OF BIOMARKERS IN POLLUTION EVALUATION}

\section{ABSTRACT}

Each day is more difficult to evaluate an ecosystem health due to the amount and diversity of xenobiotic substances introduced in them. This is why in the last two decades the use of biomarkers for the evaluation and monitoring of ecosystems as been increasingly used. A biomarker is a functional, physiological or bio-chemical response to the cellular level or molecular interactions that an organism, a population or a community reflects before a potential danger. These responses can be chemical, physical or biological. The biomarker represents an integrated signal of the pollution level of a determined area and, as a consequence, constitutes an indicator of the toxicological risk level a determined natural population can be submitted to. However, for an environmental evaluation or biomonitoring program to be effective, multiple biomarkers describing the symptoms that suggest the cause-effect relationship for a species must be used.

KEY WORDS: biomarkers, physiological responses, ecotoxicology, pollution, monitoring.

\section{INTRODUCCIÓN}

Evaluar y aminorar el deterioro ambiental hoy día es más complejo que hace 25 años atrás. Esto se debe principalmente a las consecuencias no predecibles del uso de casi 100.000 productos químicos que la sociedad moderna está produciendo en una cantidad tal, que están amenazando el ambiente (Jørgensen, 1998). Las actividades humanas se están ejerciendo en todas las direcciones, lo que contribuye cada vez más al desequilibrio de los sistemas naturales. Como producto del desarrollo industrial y el incremento de la población, el hombre ha introducido al ambiente una amplia gama de sustancias químicas que no están presentes en forma natural (Branco, 1984).

La introducción de compuestos tóxicos a los ecosistemas acuáticos, produce una variedad de respuestas en los organismos gobernadas por diferentes factores tales como: grado de toxicidad; concentración; tiempo de exposición; características ambientales del sistema receptor; edad y condición de los organismos expuestos; patógenos en el ecosistema; presencia de otros tóxicos; capacidad de biodegradación del compuesto y proceso de transporte, es decir, la distribución del compuesto químico entre la atmósfera, el agua y el suelo, dependiendo de la afinidad de dicho compuesto por cada fase (Hart y Fuller, 1974; EPA, 1982).

En las últimas décadas, la ecotoxicología ha desarrollado un nuevo enfoque metodológico basado 
en la medición de las respuestas bioquímicas, celulares, fisiológicas o comportamentales que pueden ser medidas en un organismo, a nivel de población o en una comunidad y sean reflejadas frente a un peligro potencial por uno o más contaminantes (EHC, 1993; Depledge, 1994). Cada una de estas respuestas o biomarcadores, representa una señal integrada del nivel de contaminación de una determinada zona y, por consiguiente, constituye un indicador del nivel de riesgo toxicológico al que puede ser sometida una determinada población natural (Bayne, Livingstone, Moore \& Widdows, 1976; Bayne et al., 1985; Chambers, Boone, Carr, Chambers \& Straus, 2002).

Un biomarcador se define como la variación inducida por una sustancia contaminante al nivel de los componentes bioquímicos o celulares de un proceso, de una estructura o de una función, que puede medirse en un sistema biológico (Bayne et al., 1985). Dichas variaciones pueden proporcionar información tanto sobre la amplitud de la respuesta del organismo en función de la entidad del ataque químico, como sobre la conexión entre los efectos de una sustancia contaminante y los diversos niveles de organización estructural, además pueden evidenciar efectos nocivos a muy bajas concentraciones y durante períodos cortos de exposición (Bayne et al., 1985; Palacio, 2007).

La evaluación de biomarcadores en una muestra de organismos bioindicadores de una o más zonas sospechosas de contaminación, y confrontados con organismos procedentes de una zona control, permite establecer el peligro potencial al que están sometidas la o las comunidades objeto de estudio (EULA, 1999). Además, pueden indicar el efecto ecológico de un contaminante a largo plazo en función de la exposición o no del organismo, a un nivel de contaminación que sobrepasa los niveles de normal homeostasis o compensación (Underwood y Peterson, 1988; Focardi, 1999).

\section{VENTAJAS Y LIMITACIONES}

La mayor fuerza de los biomarcadores, radica en su capacidad para evitar las serias limitaciones de las aproximaciones clásicas de la toxicología ambiental, como es la medición de residuos químicos en los organismos o en el ambiente y relacionar los efectos agudos a través de pruebas de toxicidad (Weeks, 1998). Los biomarcadores, especialmente fisiológicos y no específicos, han sido utilizados ampliamente en el laboratorio para documentar y cuantificar tanto la exposición como los efectos de los contaminantes ambientales sobre los organismos.

Para el monitoreo de exposición, los biomarcadores tienen la ventaja de reaccionar rápidamente a la exposición y así poder cuantificar únicamente los contaminantes biológicamente disponibles, y como monitores de efectos ellos son capaces de integrar los efectos de múltiples factores estresantes (Bayne et al., 1985; Weeks, 1998). En general, los biomarcadores son sensibles a los tensores y rápidamente responden, ayudando a identificar los mecanismos básicos de las relaciones causales entre un tensor y su efecto. Los biomarcadores y los bioindicadores presentan ventajas y desventajas relacionadas a su valor y uso para evaluar el efecto del estrés sobre los ecosistemas (Tabla

Tabla 1. Ventajas y limitaciones de los biomarcadores y bioindicadores. Tomado de Adams (2002).

\begin{tabular}{lll}
\hline Característica & Biomarcador & Bioindicador \\
\hline Tipo de respuesta & Subcelular, celular & Individuo a través de la \\
Indicador primario de & Exposición & Efecto comunidad \\
Sensibilidad a los tensores & Alta & Baja \\
Relación con la causa & Alta & Baja \\
Variabilidad de la respuesta & Alta & Baja - moderada \\
Especificidad al tensor & Modera - alta & Baja \\
Tiempo de respuesta & Corto & Largo \\
Relevancia ecológica & Baja & Alta \\
\hline
\end{tabular}

En la última década se ha incrementado el desarrollo de biomarcadores basados en técnicas moleculares, sin embargo, se ha cuestionado la validez de su papel ecotoxicológico para evaluar el riesgo sobre la población o el ecosistema. El mayor obstáculo para desarrollar un buen biomarcador está en medir una respuesta selectiva en un sólo organismo representante y poder extrapolar un ligero incremento o disminución del parámetro medido (un simple cambio bioquímico) a un nivel superior de organización y comprender su significado ecológico (Weeks, 1998). 


\section{CRITERIOS PARA EL USO}

Por muchos años el monitoreo ambiental se centró sobre parámetros abióticos. No obstante, la evaluación únicamente por análisis químicos se hacía difícil, debido a que los efluentes provenientes de las industrias y que entran a un cuerpo de agua, pueden contener miles de elementos individuales y compuestos que se mezclan en una o más matrices ambientales como el agua, los sedimentos o los tejidos. El criterio químico basado en los datos de laboratorio, usualmente considera solo un factor influyente en un determinado tiempo, no incluye exposiciones múltiples y son restringidas a parámetros que son convenientemente medidos. Además, el uso de solo criterios químicos para evaluar los efectos de la calidad del agua sobre los ecosistemas, puede conducir a una argumentación incompleta para la legislación como fuente de políticas, debido a que no incluye una amplia gama de mediciones ecológicas (Adams, 2002). Razón por la cual, se comenzaron a realizar monitoreos con organismos acuáticos con el fin de obtener información sobre la distribución, mecanismo de transporte, destino y efectos de los materiales antropogénicos (Bayne et al., 1985; APHA, AWWA \& WPCF, 1989). Con la ejecución de este tipo de monitoreos en ambientes acuáticos se puede (Chapman, Dexter \& Goldstein, 1987; EULA, 1999):

- Indicar a priori problemas que se desarrollan en los recursos biológicos, antes que adquieran carácter crítico.

- Dilucidar las relaciones causa-efecto.

- Establecer tendencias espaciales de la contaminación.

- Definir cambios temporales de la contaminación y su relación con consideraciones reguladoras (por ejemplo, estándares de calidad).

- Proteger las pesquerías comerciales, otros aspectos biológicos de los ecosistemas o salud pública.

- Identificar nuevos contaminantes en los ambientes acuáticos.

Existen tres estrategias para el manejo de la calidad o salud del ecosistema: 1) Regulación del efluente para limitar la descarga de sustancias peligrosas a cantidades o concentraciones que no perjudiquen los ecosistemas; 2) El Criterio de Calidad Ambiental (agua, sedimento, tejido) para limitar la ocurrencia de condiciones peligrosas en los ecosistemas; 3) Monitoreo de los efectos ambientales como un chequeo del control de la calidad, para verificar que las dos primeras $\begin{array}{llll}\text { estrategias han } & \text { (Hodson, }\end{array}$

El monitoreo abarca dos grandes objetivos: 1) describir el estado del medio ambiente, y 2) ligar la causa y el efecto donde hubo degradación. Para cumplir con esos objetivos, los programas diseñados para monitorear los efectos de los tensores pueden escogerse de una amplia gama de herramientas, desde un nivel muy específicos (biomarcadores) que ayudan al diagnóstico de la causa y del efecto, hasta bioindicadores relativamente no específicos. Teniendo en cuenta que los costos son un punto importante, y que los métodos de monitoreo deben ser aplicados repetidamente bajo condiciones estándares, se deben tener en cuenta las siguientes características con el fin de identificar biomarcadores útiles para evaluar la salud ambiental (Hodson, 2002):

1. Capacidad para definir la relación causa-efecto: se deben basar en mecanismos de efectos conocidos y con un alto grado de especificidad

2. Relevancia: los resultados deben ser relevantes para el medio ambiente y los administradores de recursos, y expresados en forma comprensible con umbrales que generen acciones de manejo.

3. Estado del desarrollo del método: los métodos deben estar lo suficientemente desarrollados e investigados para ser aplicados.

4. Expresión de los resultados: los resultados deben ser fácilmente comprendidos por el público y las autoridades ambientales.

\section{BIOMARCADORES MOLECULARES Y GENÉTICOS}

Los biomarcadores moleculares de la contaminación ambiental pueden ser definidos como macromoléculas (ácidos nucléicos, proteínas, lípidos o carbohidratos), para los cuales una alteración en su estructura o función puede ser usada como una indicación de la exposición a xenobióticos. Las respuestas bioquímicas y genéticas tales como: 1) la inducción de la citocromo P450, 2) la inhibición de la colinesterasa, 3) las metalotioneínas, 4) la vitelogenina, 5) las porfirinas, 6) el ácido retinoico y 7) la expresión génica, generalmente se basan en modelos de interacciones químicas al nivel molecular y celular, y son frecuentemente muy específicas debidas a la especificidad de los receptores de proteína por la estructura química. Como consecuencia, la especificidad de las respuestas es característica de las familias de químicos que comparten las 
mismas características. Sin embargo, la debilidad radica en la no especificidad para los efectos de una causa y la baja relevancia de los efectos ecológicos.

Las respuestas de una especie centinela expuesta en el laboratorio, pueden ser muy inconstantes debido a factores biológicos inherentes (edad, sexo, estado reproductivo), a la variabilidad genética (en términos de tasa toxicocinética, metabolismo y susceptibilidad a los xenobióticos), y a la exposición de mezclas complejas de xenobióticos y compuestos químicos naturales. Se ha trabajado mucho en peces, pero se sabe poco en invertebrados, lo cual, limita la selección de organismos indicadores, aunque ya se está utilizando la clase Theta de la glutatión S-transferasa (GST-T) como biomarcador en poliquetos. Por lo tanto, muchos de estos métodos están en etapa de investigación pues, aunque algunos biomarcadores como los enzimáticos pueden ser muy sensibles, la detección de la respuesta no necesariamente tiene implicaciones para la salud del organismo, y el comportamiento normal del biomarcador no necesariamente implica una buena calidad ambiental o su ausencia efectos nocivos (Southward, 1982; Hodson, 2002; Theodorakis y Virgin, 2002; Virgin y Theodorakis, 2002; Palacio, 2007; Rhee et al., 2007)

\section{BIOMARCADORES FISIOLÓGICOS E ÍNDICES DE CONDICIÓN}

Son biomarcadores de la salud del organismo relacionados con el estrés, entre los cuales se encuentran: 1) los hematológicos, 2) los neurotransmisores, 3) proteínas de choque térmico, 4) hormonas de estrés, 5) índices organosomáticos. Una de sus ventajas es la facilidad para colectar los datos, además, son de gran relevancia ecológica, dado que proveen de un vínculo entre el estrés fisiológico y los impactos al nivel de población. Sin embargo, debido a su inespecificidad de la causa, hay mucha incertidumbre acerca de la interpretación, y del gran número de factores ambientales y biológicos que pueden influir en la respuesta que ha generado inconsistencia entre los estudios. Por lo tanto, la capacidad de diagnóstico de las mediciones fisiológicas puede ser fortalecida por la medición de covariables (edad, sexo, tamaño, estación, hábitat, etc.) y la distribución espacial y temporal de las respuestas y los tensores (Bayne et al., 1976; Bayne et al., 1985; Widdows, 1985; Widdows y Donkin, 1991; Barton, Morgan \& Vijayan, 2002; Hodson, 2002).

\section{BIOMARCADORES HISTOPATOLÓGICOS}

Son una herramienta útil ya que pueden evaluar los efectos de exposición presentes o a priori de tensores al nivel individual, representando el nivel intermedio de organización biológica. Las lesiones detectadas en las células, tejidos u órganos representan una integración de los efectos acumulativos de los tensores fisiológicos y bioquímicos. Por lo tanto, pueden ser vinculados a la exposición, subsecuente metabolismo de contaminantes químicos, a las respuestas bioquímicas y fisiológicas tempranas y potencialmente a los niveles de organización superior. Estos cambios pueden ser detectados in situ en animales silvestres, eliminando el uso de organismos en laboratorio, lo cual tiene menos relevancia ecológica, y extrapolar los resultados a diferentes especies en su ambiente natural. Pese a que muchos factores naturales o metodológicos pueden afectar la apariencia del tejido y la diagnosis de la patología, este biomarcador puede ser útil en los monitoreos y evaluaciones ambientales si se combina con otros biomarcadores o bioindicadores, e involucra otras disciplinas como la química analítica, la inmunología, la biología reproductiva, etc. (Moore, 1985, 1988a, 1988b; Moore y Lowe, 1985; Myers y Fournie, 2002).

\section{FISIOLOGÍA ENERGÉTICA}

La evaluación de la polución ambiental, usando mediciones fisiológicas energéticas en conjunto con los análisis químicos de los contaminantes en sus tejidos, ha sido llevada a cabo con éxito en muchas situaciones de campo, al detectar y cuantificar los efectos de la polución. Estas mediciones no solo proveen de información sobre los procesos claves de adquisición, gasto y energía disponible para crecer y reproducirse, sino que también reflejan algunos de los principales mecanismos de toxicidad. La capacidad de una población para crecer y reproducirse, es decir, mantener un presupuesto energético positivo, representa un prerrequisito primario de sobrevivencia, siendo este último un criterio importante en la evaluación de la calidad ambiental. Se ha observado que la tasa de crecimiento es una respuesta fisiológica fundamental del fitness/funcionamiento de un organismo, y provee de una de las mediciones de estrés más sensitivas. 
En la evaluación del presupuesto energético se realizan mediciones que integran varios procesos fisiológicos, tales como el crecimiento potencial (CP), la relación oxígeno-nitrógeno y los índices de condición. De todas las mediciones antes mencionadas, la más utilizada es el $\mathrm{CP}$, dado que integra las respuestas fisiológicas básicas (alimentación, digestión respiración y excreción), y cualquier alteración en uno o varios de estos procesos puede afectar la energía total disponible para crecer y reproducirse. La mayor ventaja de los modelos energéticos es la integración de los efectos a través de los distintos niveles de organización y entre los tensores naturales y antropogénicos (Widdows, 1982, 1985; Widdows y Donkin, 1991; Widdows y Salked, 1992; Smaal y Widdows, 1994; Beyers y Rice, 2002; Shuhong, Huasheng \& Xinhong, 2005).

Otro biomarcador energético que fue desarrollado en 1997 es el CEA (Cellular Energy Allocation), el cual mide el balance energético en los organismos mediante la evaluación de cambios bioquímicos de la energía disponible (carbohidratos, proteínas y lípidos) y el consumo de energía (transporte de electrones y aspectos respiratorios) en la célula. Este biomarcador se ha utilizado para evaluar PAHs en anfípodos (Gammarus setosus, Onisimus litoralis) y bivalvos (Lyocima fluctuosa) y ha mostrado ser, en algunas especies, mucho más sensible que el SFG (Verslycke, Roast, Widdows, Jones \& Janssen, 2004; Coen, Robbens \& Janssen, 2006; Olsen et al., 2007).

\section{EVALUACIÓN DEL RIESGO AMBIENTAL}

Los biomarcadores han sido sugeridos como una herramienta práctica para el manejo ambiental, pero su inclusión no ha sido universalmente aceptada debido al número de preguntas sin resolver sobre su sensibilidad, utilidad y reproducibilidad. Sin embargo, en los últimos años están siendo incorporados en la evaluación del riesgo ambiental (Environmental Risk Assesment, ERA) por la legislación europea, con el fin de asegurar que los cuerpos de agua sean mantenidos o restaurados, así como garantizar la protección de los ecosistemas acuáticos de gran valor, e incluso se ha propuesto el índice de respuesta de biomarcadores (Biomarker Response Index, BRI) el cual se basa en un conjunto de biomarcadores a diferentes niveles de respuesta biológica, que puedan proveer de una medida integrada de la salud general de los invertebrados (Hagger, Jones, Leonard, Owen \& Galloway, 2006; Hagger et al., 2008).

En la evaluación del riesgo ambiental se están utilizando biomarcadores en peces tales como las enzimas Tipo I (EROD y CYP1A), productos de biotransformación (PAH en bilis y metabolitos de $\mathrm{PAH}$ en tejido), parámetros reproductivos (VTG), parámetros genotóxicos (aductos del DNA hepático) y la herramienta más moderna y poderosa, la proteómica ambiental, la cual puede indicar el modo de acción sobre las células que puede ser específico al contaminante y así proveer evidencias más directas del mecanismo químico de toxicidad (Van der Oost, Beyer \& Vermeulen, 2003; Tairova, Giessing, Hansen \& Andersen, 2009; Ricciardi, Matozzo, Binelli \& Marín, 2010; Tomanek, 2011).

\section{CONCLUSIÓN}

Los biomarcadores son de poca relevancia directa para evaluar la salud del ecosistema si no se hacen estudios de validación para demostrar la relación causal o accidental entre los diferentes niveles de organización biológica. La falta de especificidad, incluso al nivel bioquímico, significa que ningún método puede ser utilizado solo. Un programa de biomonitoreo exitoso deberá utilizar múltiples biomarcadores para describir los síntomas que sugieren la relación causa-efecto para una especie.

\section{BIBLIOGRAFÍA}

- Adams, S. M. (2002). Biological Indicator of Aquatic Ecosystems Stress: Introduction and Overview. En S. M. Adams, (Ed.), Biological indicators of aquatic ecosystems stress. (pp. 112). Maryland, USA: American Fisheries Society.

- APHA (American Public Health Association), AWWA (American Water Works Association), y WPCF (Water Pollution Control Federation). (1989). Standard Methods for the Examination of Water and Wastewater. Joint Editorial Board, USA. 1193p. 
- Barton, B. A., Morgan, J. D., y Vijayan, M. M. (2002). Physiological and Condition-Related Indicators of Environmental Stress in Fish. En S. M. Adams, (Ed.), Biological indicators of aquatic ecosystems stress. (pp. 111-148). Maryland, USA: American Fisheries Society.

- Bayne, B. L., Brown, D. A., Burns, K., Dixon,D. R., Ivanovici, A., Livingstone, D. R., Lowe,D. M., Moore, M. N., Stebbing, A. y Widdows, J. (1985). The Effects of Stress and Pollution on Marine Animals. Praeger, Greenwood Press. 384p.

- Bayne, B. L., Livingstone, D. R., Moore, M. N., y Widdows, J. (1976). A Cytochemical and Biochemical Index of Stress in Mytilus edulis. Marine Pollution Bulletin, 7, 221-224.

- Beyers, D., y Rice, J. A. (2002). Evaluating Stress in Fish Using Bionergetics-Based Stressor-Response Models. En S. M. Adams, (Ed.), Biological indicators of aquatic ecosystems stress. (pp. 289-320). Maryland, USA: American Fisheries Society.

- Branco, S. (1984). Limnología sanitaria, estudio de la polución de aguas continentales. Secretaría General de la Organización de los Estados Americanos. Programa Regional de Desarrollo Científico y Tecnológico. Washington, D.C. 115p.

- Chambers, J. E, Boone, J. S., Carr, R. L., Chambers, H. W., y Straus, D. L. (2002). Biomarkers as predictors in health and ecological risk assessment. Human and Ecological Risk Assessment, 8, 165-176.

- Chapman, P. M., Dexter, R. N., y Goldstein, L. (1987). Development of Monitoring Programmes to Assess the Long-Term Health of Aquatic Ecosystem. A Model from Puget Sound, DAME, R. F. 1996. Ecology of Marine Bivalves. An Ecosystem Approach. USA: CRC Press. 254p.

- Coen, W., Robbens, J., y Janssen, C. (2006). Ecological impact assessment of metallurgic effluents using in situ biomarker assays. Environmental Pollution, 141, 283-294.

- Depledge, M. H. (1994). The rational basis for the use of biomarkers as ecotoxicological tools. En Leonzio C., (Ed.), Nondestructive biomarkers in vertebrates. (pp. 271-295). London (UK): CRC.

- $\quad$ EHC (Environmental Health Criteria). (1993). Biomarkers and Risk Assessment: Concepts and Principles. IPCS (International Programme on Chemical Safety). EHC No. 155.

- EPA (Environmental Protection Agency). (1982). Water Quality Assessment: A Screening Procedure for Toxic and Conventional Pollutants. Part 1. Environmental Research Laboratory. Athens. 570p.

- EULA. (1999). Curso Teórico-Práctico de Postgrado: Evaluación y Monitoreo de la Contaminación Acuática. Centro EULA. Universidad de Concepción, Chile.

- Focardi, S. (1999). Contaminación antropogénica y vida marina. En Curso Teórico-Práctico de Postgrado: Evaluación y Monitoreo de la Contaminación Acuática. (pp. 1-9). Centro EULA. Universidad de Concepción, Chile.

- Hagger, J., Jones, M. B., Leonar, DR P., Owen, R. \& Galloway T. S. (2006). Biomarkers and Integrated Environmental Risk Assessment: Are There More Questions Than Answers?. Integrated Environmental Assessment and Management 2(4), 312-329.

- Hagger, J., Jones, M. B., Lowe, D. Leonard, D. R. P., Owen, R. \& Galloway T. S. (2008). Application of biomarkers for improving risk assessments of chemicals under the Water Framework Directive: A case study. Marine Pollution Bulletin 56(6),1111-1118.

- Hart Jr., C. W. \& S. L. Fuller. (1974). Pollution Ecology of Freshwater Invertebrates. Academic Press, New York. 389p.

- Hodson, P. V. (2002). Biomarkers and Bioindicators in monitoring and assessment: The State of the Art.. In S. M. Adams (ed.). Biological indicators of aquatic ecosystems stress (pp 591-619). American Fisheries Society, Maryland, U.S.A.

- Jørgensen, S. E. (1998). Ecotoxicological Research Historical Development and Perspectives. In G. Schüürmann y B. Markert (eds.). Ecotoxicology (pp 3-15). Jonh Wiley \& Sons, New York, USA.

- Moore, M. N. (1985). Cellular Response to Pollutants. Marine Pollution Bulletin. 16,134139.

- Moore, M. N. (1988a). Cellular- and Histopathological Effects of a Pollutant Gradient Summary. Marine Ecology Progress Series, 46,109-110.

- Moore, M. N. (1988b). Cytochemical Responses of the Lysosomal System and NADPHFerrihemoprotein Reductase in Molluscan Digestive Cells to Environmental and Experimental Exposure Xenobiotics. Marine Ecology Progress Series, 46,81-89.

- Moore, M. N. \& D. M. Lowe. (1985). Cytological and Cytochemical Measurements. En D. A. Brown, K. Burns, D. R. Dixon, A. Ivanovici, D. R. Livingstone, D. M. Lowe, M. N. Moore, A. Stebbing, y J. Widdows, (Eds.). The Effects of Stress and Pollution on Marine Animals. (pp. 46-74). New York: Praeger, Greenwood Press. 384p.

- Myers, M. S., y Fournie, J. W. (2002). Histopathological Indicators of Environmental Stress and Diseases Susceptibility in Fishes. En S. M. Adams, (Ed.), Biological indicators of aquatic ecosystems stress. (pp. 187-220). Maryland, USA: American Fisheries Society. 
- Olsen, G. H., Sva, E., Carroll, J., Camus, L., Coen, W., Smolders, R., Øveraas, H., y Hylland, K. (2007). Alterations in the energy budget of Arctic benthic species exposed to oil-related compounds. Aquatic Toxicology, 83, 85-92.

- Palacio, J. A. (2007). Ecotoxicología acuática. Medellín: Ed. Universidad de Antioquia. 437p.

- Rhee, J. S., Lee, Y. M., Hwang, D. S., Won, E. J., Raisuddin, S., Shin, K. H., y Lee, J. S. (2007). Molecular cloning, expression, biochemical characteristics, and biomarker potential of theta class glutathione S-transferase (GST-T) from the polychaete Neanthes succinea. Aquatic Toxicology, 83(2), 104-115.

- Ricciardi, F., Matozzo, V., Binelli, A., y Marín, M. G. (2010). Biomarker responses and contamination levels in crabs (Carcinus aestuarii) from the Lagoon Venice: An integrated approach in biomonitoring estuarine environments. Water Research, 44(6), 1725-1736.

- Shuhong, W., Huasheng, H., y Xinhong, W. (2005). Bioenergetic responses in green lipped mussels (Perna viridis) as indicators of pollution stress in Xiamen coastal waters, China. Marine Pollution Bulletin, 55, 738-743.

- Smaal, A. C., y Widdows, J. (1994). The Scope for Growth of Bivalves as an Integrated Response Parameter in Biological Monitoring. En K. J. M. Kramer, (Ed.), Biomonitoring of Coastal Waters and Estuaries. (pp. 247-267). Boca Ratón: CRC Press.

- Southward, A. J. (1982). An Ecologist's View of the Implications of the Observed Physiological and Biochemical Effects of Petroleum Compounds on Marine Organisms and Ecosystems. Philosophical Transaction of The Royal Society B: Biological Sciences, 297, 241-255.

- Underwood, A. J., y Peterson, C. H. (1988). Towards an Ecological Framework for Investigating Pollution. Marine Ecology Progress Series, 46, 227-234.

- Tairova, Z. M., Giessing, A. M. B., Hansen, R., y Andersen, O. (2009). 1-Hydroxypyrene as a biomarker of $\mathrm{PAH}$ exposure in the marine polychaete Nereis diversicolor. Marine Environmental Research, 67, 38-46.

- Theodorakis, C. W., y Virgin, I. I. (2002). Genetic Responses as Population-Level Biomarkers of Stress in Aquatic Ecosystems. En S. M. Adams, (Ed.), Biological indicators of aquatic ecosystems stress. (pp. 149-186). Maryland, USA: American Fisheries Society.

- Tomanek, L. (2011). Environmental proteomics: Changes in the Proteome of the Marine organisms in Response to Environmental Stress, Pollutants, Infection, Symbiosis and Development. Annual Review of Marine Science, 3, 373-399.

- Van der Oost, R., Beyer, J., y Vermeulen, N. P. E. (2003). Fish bioaccumulation and biomarkers in environmental risk assessment: a review. Environmental Toxicology and Pharmacology, 13, 57-149.

- Verslycke, T., Roast, S. D, Widdows, J., Jones, M. B., y Janssen, C. R. (2004). Cellular energy allocation and scope for growth in the estuarine mysid Neomysis integer (Crustacea: Mysidacea) following chlorpyrifos exposure: a method comparison. Journal of Experimental Marine Biology and Ecology, 306, 1-16.

- Virgin, I. I., y Theodorakis, C. W. (2002). Molecular Biomarkers in Aquatic Organisms: DNA Damage and RNA Expression. En S. M. Adams, (Ed.), Biological indicators of aquatic ecosystems stress. (pp. 43-110). Maryland, USA: American Fisheries Society.

- Weeks, J. M. (1998). Effects of pollutants on soil invertebrates: Links between levels. En G. Schüürmann y B. Market, (Eds.), Ecotoxicology. (pp. 645-664). New York: John Wiley \& Sons, Inc.

- Widdows, J. (1982). Field Measurement of the Biological Impacts of Pollutants. Assimilative Capacity of the Oceans for Man's Wastes. April 26-30. SCOPE/ICSU Academia Sinica, Taipei, Republic of China. pp. 111-129.

- (1985). Physiological Responses to Pollution. Marine Pollution Bulletin, 16, 129134.

- Widdows, J., y Donkin, P. (1991). Role of Physiological Energetics in Ecotoxicology. MiniReview. Comparative Biochemistry and Physiology, 100C, 65-79.

- Widdows, J., y Salked, P. N. (1992). Role of Scope for Growth in Environmental Toxicology and Pollution Monitoring. FAO/UNEP Training Workshop on: "The Techniques for Monitoring Biological Effects of Pollutants on Marine Organism". Plymouth Marine Laboratory, UK. 31p.

1. Programa de Biología, Departamento de Ciencias Biológicas, Facultad de Ciencias Exactas y Naturales, Universidad de Caldas, Colombia. 